\title{
Phenomenological Li ion battery modelling in Dymola
}

\author{
Kotub Uddin* and Alessandro Picarelli ${ }^{\dagger}$ \\ * WMG, The University of Warwick, \\ International Digital Laboratory, Coventry, CV4 7AL, United Kingdom \\ ${ }^{\dagger}$ Claytex Services Ltd., \\ Edmund House, Rugby Road, Leamington Spa, CV32 6EL, United Kingdom \\ *k.uddin@warwick.ac.uk, ’alessandro.picarelli@claytex.com
}

\begin{abstract}
In this work, the structure of a modular, acausal and reconfigurable electro-thermal battery model is described. The dynamic model structure adopted for the battery cell is based on an equivalent circuit whose parameters are generated using real cycling data through an optimisation routine written in the Modelica language. A linearised one-dimensional thermal mathematical model with lumped parameters is used to simulate temperature profiles for the cell. The cell and scaled-up pack model is parameterised for a number of commercially available cells ranging a number of cell formats, sizes and chemistries. These Dymola models are validated using highly transient and aggressive real-world as well as synthetic drive cycles.
\end{abstract}

Keywords: Lithium ion, battery, HEV, EV, PHEV, Acausal, Dymola, Modelica

\section{Introduction}

A key enabler (or constraint) of the electrified power train is the need to store energy in a form that can be easily and robustly converted into electricity. Batteries have emerged as a preferred choice in alternative energy storage but the technology still comes with significant compromises for the customer. Many of the challenges and opportunities presented by battery technology can be traced to the li-ion cell at the heart of the battery.
The need to accurately, rapidly and robustly model the performance of cells and their effects on the battery system and wider vehicle is of paramount importance to vehicle OEMs. While multiple modelling approaches are available for Li-ion cells, a balance is required to produce a model than has the flexibility to map the microscopic scale effects of internal cell mechanisms to the macroscopic scale of pack and vehicle dynamics in a timely and cost effective manner.

Moreover it is important for any of these battery models to be readily integrateable with an existing electrified powertrain and control simulation toolset, where an acausal simulation structure (as opposed to input-led) can be advantageous. In this paper, we extend the INEEL FreedomCar program model [1] to include temperature dependence, voltage hysteresis, self-discharge and diffusion limitation. We present a generic routine that can be used to generate model parameters based on optimisation [2]. Moreover, we utilise the capacity of Modelica to avoid assignment statements and base our model on equations so as to achieve acausality. Finally, we show an efficient method for scaling-up the cell electro-thermal model, while maintaining the ability to uniquely parameterize individual cells, to pack level without substantially compromising simulation time.

\section{Model development}

\subsection{Cell Model}


Modelling the electronic and thermal behaviour of a battery cell requires characterization of mechanisms across multiple time domains and model parameters that are dynamically interlinked. The equivalent circuit model (ECM) adopted in this work is shown in Figure 1 and consist of a parallel RC network connected in series with a capacitor, a resistor and an ideal voltage source. As shown in Figure 1, the circuit is mainly composed of three parts including an open-circuit voltage source $V_{o c}$, internal resistances and equivalent capacitances. The internal resistances include the ohmic resistance $R_{0}$ which comprises all electronic resistances and the polarization resistance $R_{d}$ which when coupled with $C_{d}$ accounts for ion diffusion. The equivalent capacitance $C_{T h}$ is used to describe the transient response during charging and discharging [1].

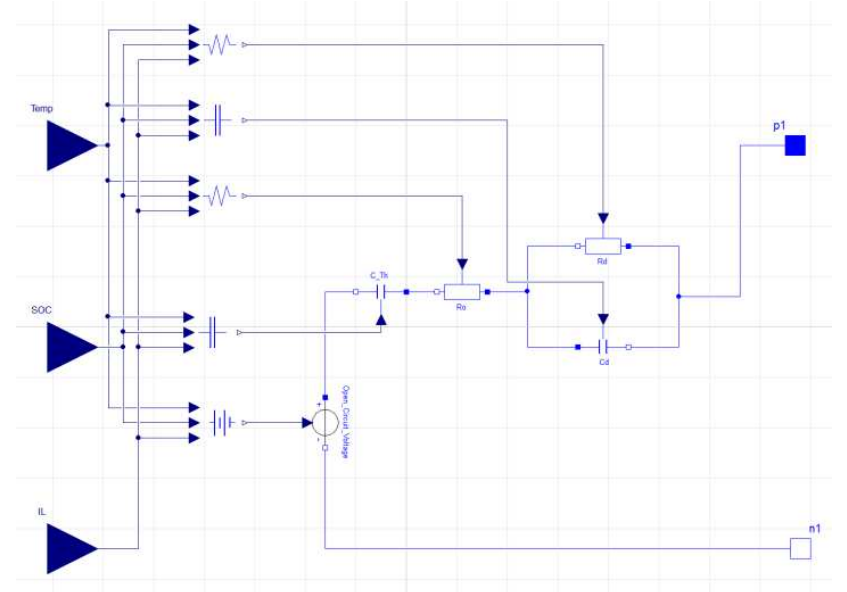

Figure 1: Depicting the equivalent circuit model of a Li-ion battery system. The circuit represents temperature, state of charge and current dependency of the circuit components; selfdischarge and the hysteresis effects added with open circuit voltage.

The electrical behaviour of the ECM shown in Figure 1 is given by [1]:

$$
\begin{aligned}
V_{L}=V_{O c}-I_{L} R_{0}- & U_{T h}-U_{d} \\
\frac{d}{d t} U_{d}+\frac{U_{d}}{R_{d} C_{d}} & =\frac{I_{L}}{C_{d}} \\
\frac{d}{d t} U_{T h} & =I_{L} \frac{d V_{O C}}{d Q}
\end{aligned}
$$

where $V_{L}$ is the terminal voltage, $I_{L}$ is the load current, $U_{T h}$ is the voltage drop across the capacitor $C_{T h}, U_{d}$ is voltage drop due to polarisation effects and $Q$ is accumulated charge; the mentioned variables are time dependent. This coupled set of equa- tions (1) can be solved analytically and without loss of generality the solution is given by:

$$
\begin{aligned}
V_{L}=V_{o c}-I_{L} R_{0} & \\
& -\frac{1}{C_{T h}} \int_{t} I_{L} d t \\
& -\frac{e^{-\frac{t}{R_{d} C_{d}}}}{C_{d}} \int e^{\frac{t}{R_{d} C_{d}}} I_{L} d t
\end{aligned}
$$

The last term on the right hand side arises from the $\mathrm{RC}$ component and is decoupled from contributions from the other components in the circuit. This suggests that from a mathematical viewpoint introducing more RC terms into the ECM will not lead to challenging parameterization algorithms, however, in so doing one must give attention to balancing the computational effort with accuracy yield. In this work we consider a single (modified) RC circuit, similar to that proposed by INEEL FreedomCar program [2], and will show that this leads to sufficient accuracy.

Equation (2) can be re-written in a closed form thus [2]:

$$
\begin{gathered}
V_{L}=V_{o c}-I_{L} R_{0}-\frac{1}{C_{T h}} \int_{t_{0}}^{t_{f}} I_{L}(t) d t-I_{P}(t) R_{d} \\
I_{P, i}=\left[1-\frac{1-\exp \left(-\frac{\Delta t}{\tau_{P}}\right)}{\frac{\Delta t}{\tau_{P}}}\right] I_{L, i} \\
+\left[1-\frac{1-\exp \left(-\frac{\Delta t}{\tau_{P}}\right)}{\frac{\Delta t}{\tau_{P}}}\right. \\
\left.-\exp \left(-\frac{\Delta t}{\tau_{P}}\right)\right]_{P, i} \\
+\exp \left(-\frac{\Delta t}{\tau_{P}}\right) I_{P, i-1}
\end{gathered}
$$

which is written in matrix form:

$$
\left(\begin{array}{c}
V_{L, 1} \\
V_{L, 2} \\
\vdots \\
\vdots \\
\vdots \\
V_{L, n}
\end{array}\right)=\left(\begin{array}{cccc}
1 & I_{L, 1} & A h_{L, 1} & I_{P, 1} \\
1 & I_{L, 2} & A h_{L, 2} & I_{P, 2} \\
\vdots & \vdots & \vdots & \vdots \\
\vdots & \vdots & \vdots & \vdots \\
\vdots & \vdots & \vdots & \vdots \\
1 & I_{L, n} & A h_{L, n} & I_{P, n}
\end{array}\right)\left(\begin{array}{c}
V_{o c} \\
R_{0} \\
C_{T h}{ }^{-1} \\
R_{d}
\end{array}\right)
$$


where

$$
A h_{L, i}=\sum_{k=0}^{i} I_{L, k} \Delta t
$$

Solving equation (4) via an optimisation routine in Modelica, which constitutes a part of the battery library, generates estimates for the parameters of the ECM at a fixed temperature $(T)$, load current and state of charge $(\mathrm{SoC})$ defined by

$$
\operatorname{SoC}=\operatorname{SoC}\left(t_{0}\right)-\frac{100}{Q_{\text {rated }}} \int_{t_{0}}^{t_{f}}\left(I+S_{D}\right) \cdot d t
$$

where $Q_{\text {rated }}$ is the rated capacity of the cell (the total amount of charge that can be reversibly cycled from the cell) and $S_{D}$ is the self-discharge contribution given by [3]:

$$
S_{D}=k_{0}\left(-\frac{E_{A}}{R_{g} T}\right) S o C
$$

where the ratio of activation energy to the molar gas constant $E_{A} / R_{g}$ is determined through observations and on timescales of a few hours can be taken to be zero. The optimisation routine runs a sweep for $\tau$ within a range of values. The value of $\tau$ which yields the least error when predicting $V_{L}$ via equation 4 is selected for the reference data set.

The optimisation routine is repeated for various temperatures, current pulses and $S o C$ values to produce a three dimensional map of battery parameters as a function of $S o C, I_{L}$ and $T$. These parameters are then fed into the equivalent circuit models.

The Hysteresis contributions $V_{h}$ to cell voltage is modelled by the following first-order differential equation which we couple to open circuit voltage $V_{o c}$ [4]:

$$
\frac{\partial V_{h}}{\partial t}=-\beta\left(I_{L}-\epsilon S_{D}\right)\left[V_{h, \max }+\operatorname{sign}(I) V_{h}\right]
$$

where the constants $\beta$ and $\epsilon$ are to be determined and $V_{h, \max }$ is the limiting hysteresis voltage. Equation (8) is constructed such that for prolonged as well as large pulse charge currents the hysteresis voltage tends to $V_{h, \max }$ while for prolonged as well as large pulse discharge currents the hysteresis voltage tends to $-V_{h, \max }$. Moreover, if there is a prolonged period of zero current the hysteresis voltage tends to $-V_{h, \max }$ through the self-discharge effect.

The $R C$ component shown in Fig 1 attributes a time constant $\tau=R_{d} C_{d}$ to the bulk diffusional process of $\mathrm{Li}$ ions in the solid phase. We account for diffusion limitation, where the surface concentration of lithium may be significantly different than the average concentration contained within the active material particle, by allowing the time constant to be a function of current and assume the following simple power-law form [5]:

$$
\tau=\left|a_{0}+a_{1} I_{L}+a_{2} I_{L}^{2}+a_{3} I_{L}^{3}+O\left(I_{L}^{4}\right)\right|
$$

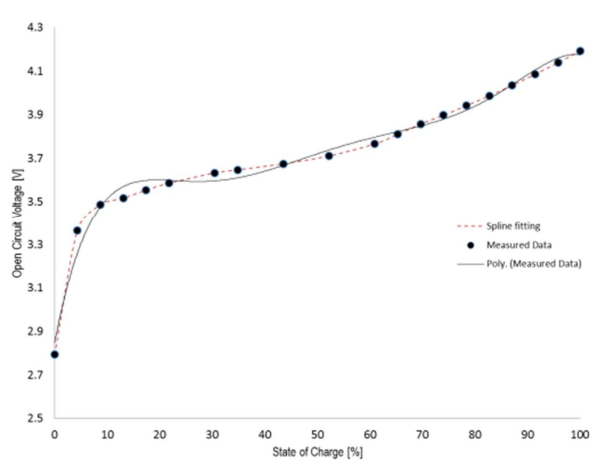

Figure 2: A comparison of $V_{o c}$ estimation using polynomial fitting $\left(6^{\text {th }}\right.$ order) and spline functions applied to $V_{o c}(S o C)$ values generated through solving Eq. (4).

To generate mathematically smooth estimates for the battery parameter $\left(Y=Y\left(S o C, I_{L}, T\right)\right)$ the usual practice is to use polynomial fitting functions. This method has the disadvantage that it often does not fit the data well as in the case of Open Circuit Voltage depicted in Fig. 2. In this work we utilize spline functions to generate smooth estimates for $Y(S o C, T)$ which avoid oscillations in interpolated values either side of outliers. We find that while both estimates follow the general trend of the data in the highly non-linear regions of the curve the accuracy of the spline function is much greater compared to a polynomial fit.

Cubic splines are preferred over lower degree splines. With first-degree splines the slope of the spline may change abruptly at the knots (i.e., data points) and for second-degree splines the discontinuity is in the second derivative which means that the curvature of the quadratic spline changes abruptly at each node. The cubic spline function $S$ is de- 
fined in the $\mathrm{X}$ interval $\left[X_{0}, X_{f}\right]$ such that $S$ is a polynomial of degree at most 3 on each subinterval $\left[X_{i}, X_{i+1}\right]$ and $S$ is continuous up to its second derivative.

Spline interpolation is coupled with linear interpolation where there is a weak correspondence between a variable $\left\{S o C, I_{L}, T\right\}$ and the model parameter $Y=$ ter $\left.Y=Y\left(S o C, I_{L}, T\right)\right)$.

A further improvement in stability was achieved using Akima splines. The calculation of the derivative only relies on data from local points, hence reducing the amount of oscillations between data points in the interpolation.

Temperature is modelled as lumped value. For modelling Li-ion batteries this is convenient because of the resulting simplicity of the governing equations. Such assumptions can be suitable if temperature gradients within the cell body are negligible [5] which does not hold for most HEV applications were currents are large. It has recently been shown that a cell with a temperature gradient maintained across is has a lower impedance than one held at the theoretical average temperature [6]. Our assumption therefore will introduce some errors in voltage predictions but this, as will be demonstrated, can be negligible. The thermal model for a single cell is depicted in Figure 3 where the heat generated in [J] is given by:

$$
\dot{Q}=I\left(V-V_{O C}-T_{r e f} \frac{d V_{O C}}{d T}\right)
$$

where a dot represents a time derivative and $I\left(V-V_{O C}\right)$ represents irreversible joule heating caused by Li-ion transport. The last term on the right hand side of Eq. (9) represents the reversible rate of heat generation due to entropy change. This model assumes no specific geometry for a single cell beyond a total volume with uniform temperature for some arbitrary body.

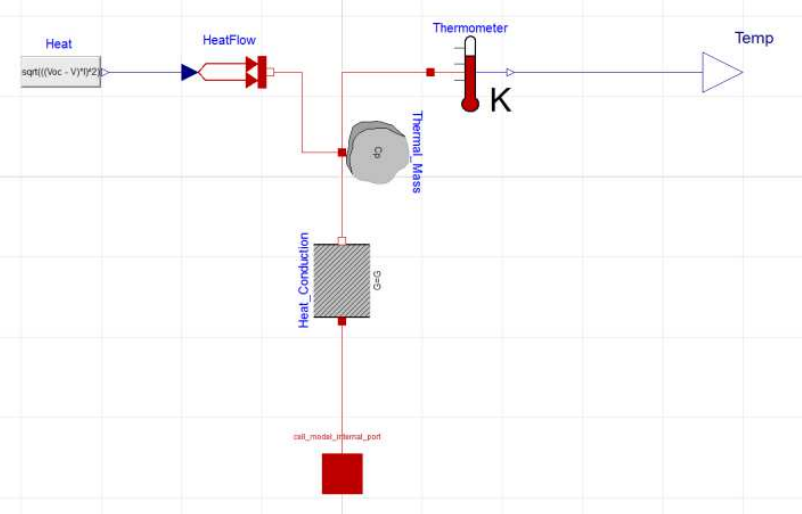

Figure 3: Figure depicting the 1D thermal model which is coupled to the electrical model via the heat source (equation 9).

\subsection{Pack Model}

The pack model consists of an electrical network and a thermal network connecting electrical and thermal paths respectively.

\section{Pack electrical model}

A pack is constructed from modules which are constructed from sub-modules. The sub-modules are of two types: only parallel connections and only series connections. This allows us to make effective "cells" which become the building blocks of our model. The sub-modules are constructed by appropriately looping electrical connections of a single cell. Each cell is then allowed to be unique, i.e., take unique values of initial $S o C, \mathrm{C} / 3$ discharge capacity, internal resistance, polarisation resistance, $V_{O C}$, bus bar connection resistance - through a data table. Such uniqueness of cell parameters allows for various interesting studies including cell balancing, diagnostics, performance limitations, design optimisation and so forth. The sub-modules are either connected via parallel or series loops to construct modules which can be connected to construct a pack. The unspecified parameters of the pack electric model include bus-bar resistance and inter-module connection resistance. For studies of high frequency ripple, the inductance of bus bars can be included as a parameter; however, in what is presented here this option is deselected.

The advantages of using component arrays over separate instantiations of components is firstly: less model diagram layer space being taken up, but more importantly: the ability for the model to be scaled up 
or down without having to manually redefine the architecture.

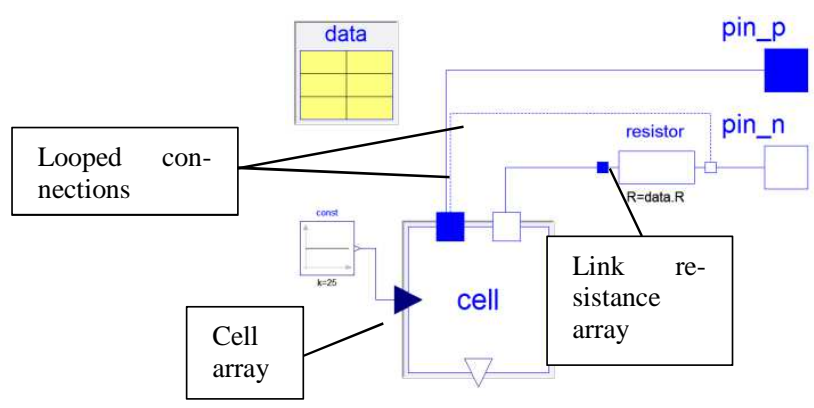

Figure 4: Dymola model diagram layer showing component array methodology for scalable stacks.

In the example shown in Figure 4, each element in the cell and resistance arrays $(\operatorname{size}=n)$ have been linked using the following notation:

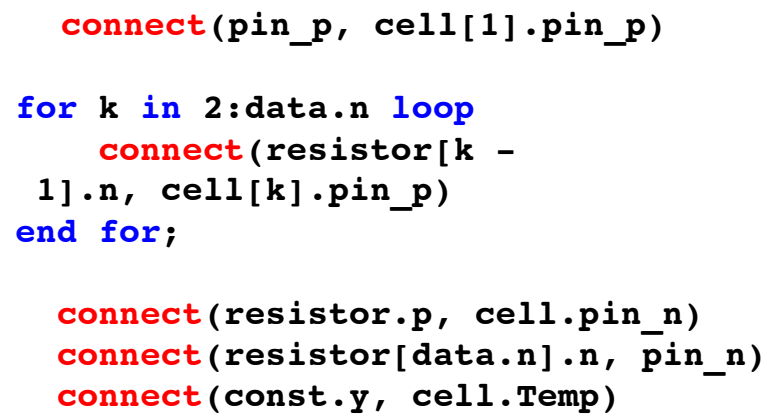

Symbolic Manipulation is a powerful tool used to simplify the systems of equations generated for a model during compilation. The model equations are rearranged into a form where the unknowns can be calculated whilst the redundant equations are removed. Dymola like other Modelica based tools has its own version of Symbolic Manipulation which helps achieve time-effective model computation.

The simplification of the systems of equations leaves the accuracy of the model intact whilst dramatically reducing the computational effort required to solve the original model equations [7].

\section{Pack thermal model}

Akin to the electrical pack model we connect thermal paths between cells via heat-ports. Thus, employing loops we thermally connect cells within submodules, sub-modules within modules and modules within a pack which is attached to a global cooling circuit. In our work we consider two cooling models:

\section{Control based ideal cooling system}

This simple model mimics an ideal cooling strategy. The cooling system remains inactive as long as the temperature of the cells in the pack remains below some critical temperature $T_{c}$ (a design parameter). As soon as the temperature exceeds $T_{c}$ the cooling system takes action: it extracts heat $Q(T)$ [W] away from every cell in the pack uniformly. The tion $Q(T)$, which is a function of cell temperature, can take any form and in the simplest design will be a constant (i.e., regardless of how hot the cells are, the cooling system exerts the same effort). Once the temperature is forced below $T_{c}$ the cooling system will return to its idle state.

\section{Fluid based Cooling Model}

This model couples simple fluid dynamics with the existing electro-thermal model. This model connects every cell in the pack via their sub-modules and modules to the global cooling circuit via cooling plates. The surface area of the cooling plate is a critical feature and is a parameter of the model. The cooling system is traversed by coolant (incompressible mixture of water and ethylene glycol, the ratio of which is a parameter of the model) that is forced by an ideal pump with a mass flow rate $m_{\text {flow }}$ that is a parameter of the model.

\section{Validation}

We have parameterized our model for a number of commercially available cells ranging a number of cell formats, sizes and chemistries including cells with Lithium iron phosphate (LFP), Manganese spinel (LMO) and Lithium nickel manganese cobalt (NMC) cathode materials and graphite and Lithium titanate (LT) anode materials. We find cells with the same chemical compositions have similar $V_{O C}(S o C)$ profiles; however, internal resistance - which is influenced by factors such as electronic contact between active electrode materials and current collectors, homogeneity of the active material paste, the battery internal structure - and polarisation re- 
sistance - influenced by electrolyte composition are found to differ. It can thus be established that the manufacturing process itself will have a bearing on a batteries performance characteristics.

In what follows of this sub-section we present some validation results for the electro-thermal cell model. While the model has been validated for a number of commercially available cells here we present results for a 20Ah LFP pouch cell. The cells were cycled and monitored using a Bitrode MCV 16-100-5 EV/HEV Battery Cell Test System.

\subsection{Cell level validation}

The validation of the 20Ah LFP cell utilised an aggressive artificial cycle, shown in Figure 5a, in order to test the model boundaries. The subsequent results of the electro-thermal model are shown, with laboratory test data, in Figures 5b-5c.

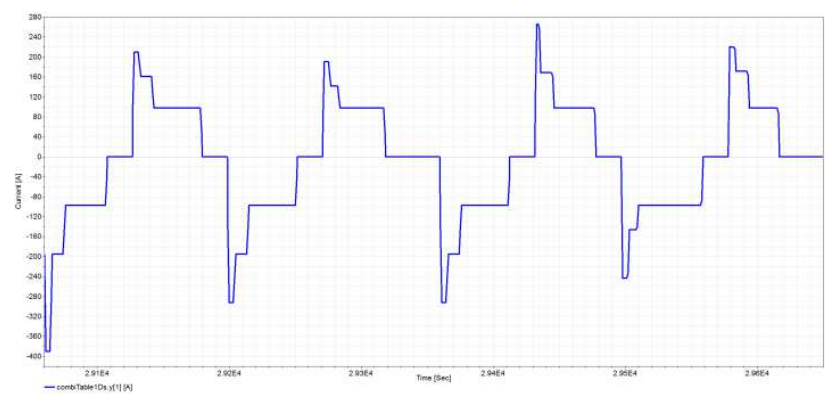

Figure 5a: Depicts a highly demanding current profile used for this validation process. The duration of this cycle is 600 seconds (excluding rest time) and the pulses range between 20C discharge and 10C charge.

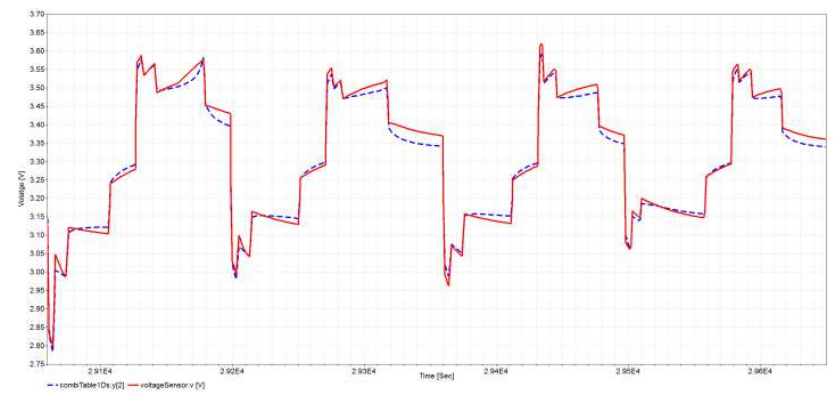

Figure 5b: A comparison of predicted terminal voltage using the cell model (blue line) with actual test data (red line) for a $20 \mathrm{Ah}$ LFP cell. The maximum transient error is less than $50 \mathrm{mV}$.

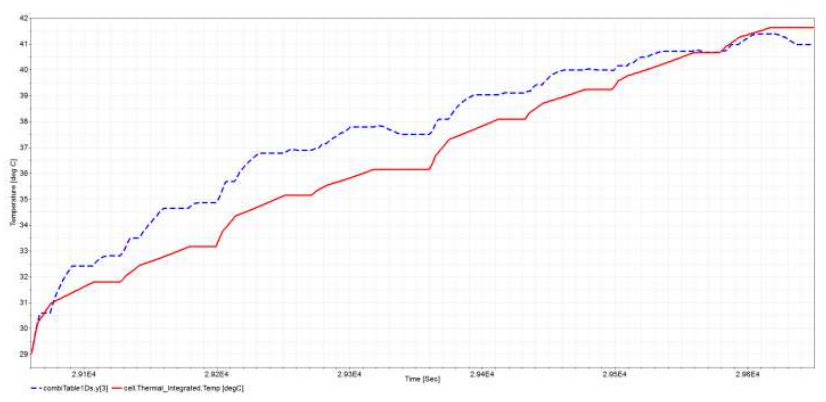

Figure 5c: A comparison of temperature predictions using the reduced order model (red line) with laboratory data (blue line) for an A123 20Ah LFP cell.

\subsection{Pack level validation}

For pack validation we use a commercial pack comprising 214 20Ah LFP cells in a 2p107 configuration. The cells are organised into 5 modules: 4 modules with $2 \mathrm{p} 24 \mathrm{~s}$ configuration and a final module with a $2 \mathrm{p} 11$ s configuration. There is a service break that splits the pack between 2 modules and 3 modules $(2 \mathrm{p} 48 \mathrm{~s}$ and $2 \mathrm{p} 59 \mathrm{~s})$ for safety. The cells are welded to the bus bar by laser welding and the modules are connected via thick $48 \mathrm{~mm}$ cables with a resistance of $240 \times 10-8240 \times 10^{-8} \Omega$ and inductance of $50 \mathrm{nH}$. The pack was cycled and monitored using a Bitrode FTF-500-900 EV/HEV Battery Pack Test System and temperature was recorded using t-type thermocouples connected to a pico logger.

The pack was cycled with a number of PHEV cycles. Here we present results of a single charge sustaining mode cycle. It is worth mentioning that the nominal transient discrepancy between modelled and measured voltages were below $2 \%$ during most part of the simulation and in some cases peaked to $5 \%$ as shown in Figure $6 \mathrm{~b}$ (i.e., the maximum transient discrepancy between modelled and measured voltage is less than 5\%). The pack was connected to a cooling system passing liquid coolant at a rate of $27 \mathrm{~L} / \mathrm{min}$ thorough the cooling plates attached to the bottom of the pack (opposite end to cell tabs). 


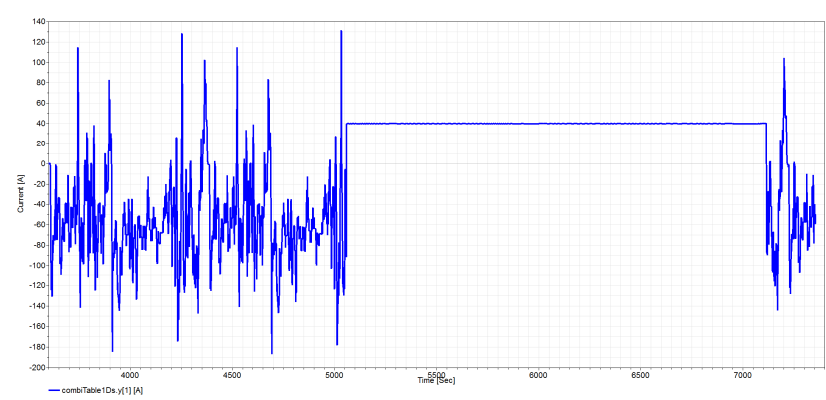

Figure 6a: A 63 minute current cycle consisting of theree phases: The first 24 minute charge depleting phase with current between $-200 \mathrm{~A}<\mathrm{I}<130 \mathrm{~A}$ takes the cell from $70 \%$ SoC down to $10 \%$ SoC; this is followed by a 34 minute phase of constant $40 \mathrm{~A}$ charge which takes the cell back up to $70 \%$ SoC; the final phase is a 5 minute charge depleting phase with similar current magnitudes.

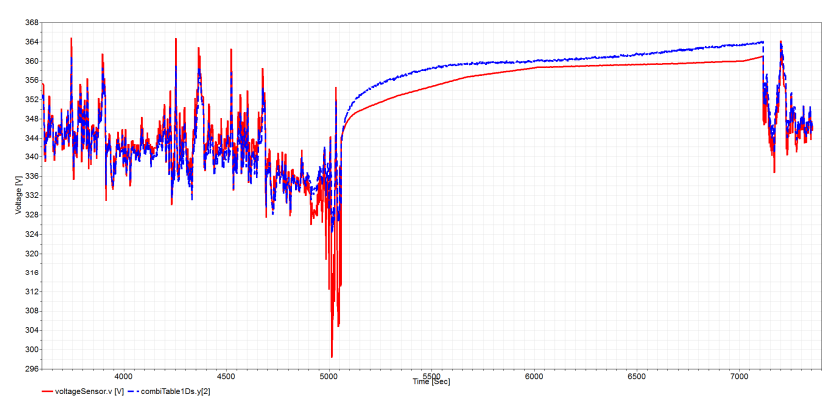

Figure 6b: Shows a good fit between modelled voltage (red) and measured voltage (blue). During most of the simulation errors were below $2 \%$, with a peak error of $5 \%$ at around the $5000 \mathrm{sec}-$ onds.

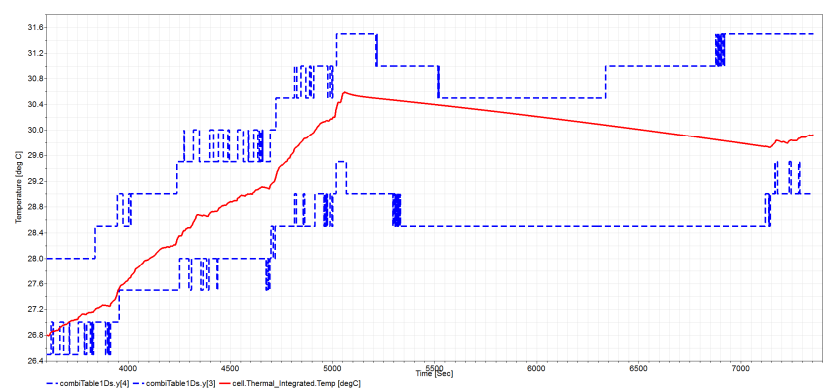

Figure 6c: Compares modelled temperature (red line) verses maximum recorded cell temperature in the pack and minimum recorded temperature (blue dashed lines).

\section{Conclusions}

In this paper we present a library of models that extend to construct a coupled, dynamic, electro-thermal model of battery cells and packs. At the elementary level we utilize a single polarization equivalent circuit model (ECM) to capture the Ohmic and diffusional characteristics of a Lithium ion battery. The EC model is then developed to include effects of hysteresis, self-discharge and diffusion limitation.
A distinguishing property of this model is the inclusion of diffusion limitation effects through a current dependant time constant. This property better mimics the solid diffusional dynamics of $\mathrm{Li}^{+}$intercalation into the active material.

Employing an optmimisation routine we extract state of charge (SoC), temperature (T) and current dependent (IL) model parameters from High Pulse Power Characterization (HPPC) data. This extracted data then forms a three dimensional "look-up table" which is interpolated using Spline functions in the ranges $0 \% \leq \mathrm{SOC} \leq 100 \%$ and $-20^{\circ} \mathrm{C} \leq \mathrm{T} \leq 65^{\circ} \mathrm{C}$.

The model (cell, module and pack) is acausal and thus utilises physical pins that mimic battery terminals. Stimuli are therefore any load acting on the battery via the tabs (as is the case in reality) and outputs are any measurements that are made across the tabs (using modelled sensors).

The cell model was validated using a number of commercially available cells. Our combined validation results showed a maximum of $50 \mathrm{mV}$ discrepancy between measured and tested voltages at cell level and a $1.4^{\circ} \mathrm{C}$ discrepancy between measured and tested temperature at cell level.

Our pack model is constructed from a series-parallel configuration of cells where cell-to-cell connections are modelled by a resistor. Our focus for this integrated architecture was methods of automated and unique parameterization which facilitates, for example, the study of the effects of SoC imbalance, anomalous resistances and SoC balancing mechanism.

An important characteristic of the model presented here, which is unique to Modelica code, is that it is based on equations instead of assignment statements. The main advantage is that the solution direction of equations will adapt to the data flow context in which the solution is computed. The nature of the equation based approach means that the models are acausal. This in turn means that the modeler does not have to rewrite or rearrange the system equations when using the components in different scenarios, for example forward and inverse dynamic modelling situations. More advantageously a model of a battery behaves physically like battery. So for example, if a resistor is connected to the physical pins (modelled negative and positive terminals of a battery) the battery will discharge causing a rise in temperature, fall in SOC, fall in voltage and so on. 
In this work we demonstrate Dymola's ability for multi domain modelling. We find that the Mdoelica libraries including Electric, Fluid and Thermal readily facilitate the construction of coupled electrothermal battery models. However, we also find that Dymola is lacking in some areas of numerical problem solving, particularly in solving coupled nonlinear simultaneous equations as well as coupled non-linear partial differential equations. We circumvent these problems by employing regression methods for predicting battery parameters.

\section{References}

[1] H. He, R. Xiong, J. Fan, Evaluation of lithiumion battery equivalent circuit models for state of charge estimation by an experimental approach, Energies, 4 (2011) 582-598.

[2] I.N.E.E. Laboratory, Battery Test Manual for Plug-In Hybrid Electric Vehicles, in, Assistant Secretary for Energy Efficiency and Renewable Energy (EE),

Idaho Operations Office: Idaho Falls, ID, USA, 2010., 2010.

[3] M.W. Verbrugge, R.S. Conell, Electrochemical and thermal characterization of battery modules commensurate with electric vehicle integration, Journal of the Electrochemical Society, 149 (2002) A45-A53.

[4] M. Verbrugge, E. Tate, Adaptive state of charge algorithm for nickel metal hydride batteries including hysteresis phenomena, Journal of Power Sources, 126 (2004) 236-249.

[5] K. Uddin, A. Picarelli, C. Lyness, N. Taylor, Acausal electro-thermal Li-ion battery models for automotive applications, Journal of Power Sources, to be published (2014).

[6] Y. Troxler, B. Wu, M. Marinescu, V. Yufit, Y. Patel, A.J. Marquis, N.P. Brandon, G.J. Offer, The effect of thermal gradients on the performance of lithium-ion batteries, Journal of Power Sources, 247 (2014) 1018-1025.

[7] P.A. Fishwick, Handbook of dynamic system modeling, CRC Press, 2007. 